\title{
A Population-Based Outcomes Analysis of the Impact of Age on Morbidity and Mortality Following Gastrectomy: An Analysis of 13,799 Patients from the Nationwide Inpatient Sample Database
}

\author{
Ami Karkar ${ }^{1}$, Sachin Patil ${ }^{1}$, Ronald S. Chamberlain ${ }^{1,2,3^{*}}$ \\ ${ }^{1}$ Department of Surgery, Saint Barnabas Medical Center, Livingston, USA; ${ }^{2}$ Saint George’s University School of Medicine, St. \\ George, Grenada; ${ }^{3}$ Department of Surgery, University of Medicine and Dentistry of New Jersey, Newark, USA. \\ Email: *rchamberlain@barnabashealth.org
}

Received August 29 ${ }^{\text {th }}$, 2012; revised September $30^{\text {th }}$, 2012; accepted October $12^{\text {th }}, 2012$

\begin{abstract}
Introduction: Gastric cancer is the $4^{\text {th }}$ most common malignancy and second leading cause of cancer-related death worldwide, both its incidence and mortality have decreased over the past 70 years. Advancing life expectancy, as well as subtle change in the type and location of gastric cancer in the US, has resulted in an increased number of elderly patients requiring gastric surgery. By 2050, the population older than 85 years is projected to reach 20.9 million, and as a result, the need to assess the operative outcomes and mortality following gastrectomy in this group is imperative. This study sought to assess age-related clinical outcomes following gastric cancer surgery across all age groups to provide more precise data for pre-operative surgical risk stratification. Methods: Discharge data on 40,276,240 patients was collected from Nationwide Inpatient Sample Database (NIS) (2004-2008). Data on patients undergoing gastrectomy as the primary procedure was analyzed including age, gender, elective/non-elective admission, pre-operative length of stay (LOS), total LOS, and mortality. Eight age groups were compared for two procedures: total gastrectomy (TG) and partial gastrectomy (PG). Categorical data was compared using the Chi square test and continuous data using the Student's $t$ test. Univariate analysis and multivariate regression analysis were performed to assess independent variables. Results: 13,799 patients underwent gastrectomy surgery with $23.7 \%$ having TG and 76.3\% PG. Gastric carcinoma was the most common indication for TG, while benign gastric disease was more common for PG, especially in years $51-70$ ( $p<$ 0.001).The mean age for TG and PG groups were $63 \pm 12.8$ and $64 \pm 15$ years respectively. Males underwent twice the number of TGs $(p<0.001)$, whereas equal number of males and females underwent PG $(p<0.001)$. The number of TGs increased over the 5-year study period, with the highest \% change noted in those 41 - 50 years (1500\%). PGs performed decreased overall, especially in patients $<60$ years, however PGs increased in patients $>81$ years with the greatest $\%$ change in the oldest patients $>91$ years $(13 \%)$. Non-elective admissions were more common for PGs $(\mathrm{N}=4844$, 41\%) than TGs $(\mathrm{N}=695,21.2 \%)$. Mean pre-operative LOS and total LOS increased with advancing age for both TG and PG $(p<0.001)$. HTN $(45 \%)$, electrolyte imbalances $(28 \%)$ and chronic pulmonary disease $(18 \%)$ were the most prevalent co-morbidities and significantly affected mortality on univariate analysis $(p=0.001)$. Respiratory $(18 \%)$ and GI complications (11\%) were the most common post-operative complications following TG, while GI (9.6\%) and bile duct fistulas (7.2\%) were most common after PG. Overall TG and PG mortality rates were $7.6 \%$ and $6.4 \%$ respectively. Mortality increased with advancing age in both groups $(p<0.001)$. Multivariate analysis identified HTN, valvular disease, anemia, malignancy and non-elective admissions as independent predictors of mortality $(p=0.001)$. Conclusions: Advancing age is not an independent predictor of mortality following gastric surgery. Gastrectomy for gastric cancer is associated with a higher mortality than for benign gastric diseases. Non-elective admission, and pre-existing hypertension, valvular disease and anemia independently predicted increased morbidity and mortality following gastric surgery and should be carefully considered in surgical planning and counseling. Gastric carcinoma is the most common indication for TG, while benign gastric disease is a more common indication for PG. The number of TGs performed is increasing, especially in the males and younger patients, and may reflect an increased incidence of body and cardiac lesions.
\end{abstract}

Keywords: Gastric Cancer; Gastrectomy; Partial Gastrectomy; Total Gastrectomy

"Corresponding author. 


\section{Introduction}

Gastric cancer is the fourth most common malignancy and second leading cause of cancer-related death worldwide. Gastric cancer primarily presents in advanced stages especially among the elderly (>80 years) and is associated with a poor outcomes unless diagnosed early [1]. Hazanaki et al. observed advanced gastric cancer in $56 \%$ of patients $>80$ years compared to $27.9 \%$ of patients $<80$ years old $(p<0.01)$. Overall in-hospital mortality ( $2 \%$ versus $0 \%$ ), and 5 -year overall survival rates $(46.1 \%$ versus $71.1 \%(p<0.01))$ were substantially higher among those $>80$ years compare those $<80$ years [2]. Precise explanations for the higher incidence of advanced disease and worse outcomes in the elderly remains largely unknown but is likely related to life expectancy, pre-existing co-morbidities, as well as age-related differences in tumor location and histology [3,4]. Complete surgical resection with a R0 margin provides the highest survival benefit for gastric cancer, however this is achievable in only a select group of patients (approximately $50 \%$ ). Moreover, gastrectomy is associated with significant morbidity and mortality, particularly in the elderly, and in-hospital mortality rates as high as $16.6 \%$ after total gastrectomy (TG) and $15.3 \%$ after partial gastrectomy (PG) have been reported [5]. The percent of patients over 80 years is the fastest growing segment of the US population, and by 2050, those over 85 years is projected to reach 20.9 million. As a result, there is a growing and important need to have precise information related to the operative risks, outcomes and mortality following gastrectomy in elderly patients in order to improve surgical decision making and patient counseling. This study sought to compare population-based outcomes following gastrectomy across a wide range of age groups to determine whether advancing age, or other factors, were independently associated with increased morbidity and mortality.

\section{Methods}

Discharge data for 40,276,240 patients from the 20042008 Nationwide Inpatient Sample Database (NIS), a part of the Healthcare Cost and Utilization Project (HCUP) of the Agency for Healthcare Research and Quality (AHRQ) was analyzed. The NIS involved no identifying patient information and was thus exempt from Institutional Review Board approval at our institution. Information on patients who had undergone total gastrectomy (ICD 9 code: 439.1) and partial gastrectomy (ICD 9 codes: 438.1 and 438.9) as the primary procedure was abstracted. Eight age groups (in increments of 10 years starting at 21 years) were compared for two procedures: total gastrectomy (TG) and partial gastrectomy (PG). The number of patients, gender ratio, most com- mon primary diagnosis, non-elective nature of admission, co-morbidities, pre-operative length of stay (LOS), total length of stay (LOS), and morbidity and mortality rates were abstracted separately for both TG and PG. Information on complication rates was extracted using ICD-9 codes. The type of complications and their broad grouping is detailed in Table 1. In addition, the total number of procedures performed (both TG and PG) over the five year period, as well as the percentage change in the number of procedures for each type of gastrectomy (Figures $\mathbf{1}(\mathbf{a})$ and (b)), were calculated by dividing the number of gastrectomies performed in 2008 by the number gastrictomies performed in 2004.

Statistical analysis was performed using the Chi square test for categorical data and the Student's t test for continuous data. A value of $p<0.05$ was considered statistically significant. Univariate analysis and multivariate logistic regression analysis were performed to assess factors affecting mortality following gastrectomy. Data analysis was performed using SPSS version 19.0 (SPSS, Inc). As per NIS database reporting guidelines, values $<10$ are not shown in the results section and in the tables.

\section{Results}

\subsection{Demographic Information}

13,799 patients identified in the NIS underwent either total gastrectomy (TG) or partial gastrectomy (PG) between 2004 and 2008, and formed the study population. Clinicopathological characteristics are detailed in Table 2. $23.7 \%(\mathrm{~N}=3271)$ patients underwent a $\mathrm{TG}$, while $76.3 \%(\mathrm{~N}=10,528)$ underwent a PG. The largest number of TG were performed in patients age $61-70$ years $(\mathrm{N}=$ 980, 30\%), whereas the largest number of PG were performed in those age 71 - 80 years $(\mathrm{N}=2436,23.1 \%)$. The mean age for the TG and PG groups were $63.1 \pm$ 12.8 and $64.1 \pm 15$ years, respectively. Twice the number of males compared to females received a TG $(p<0.001)$, whereas equal numbers received a PG $(p<0.001)$. The greatest gender difference was observed among those 61 70 years old for the TG group (M:F, 2.9:1), and among those 21 - 30 for the PG group (M:F, 1:1.6). Figures 1(a) and (b) detail the change in number and percent of TG and PG performed during the study period. TGs increased over the 5-year study period, with the highest percentage change among those 41 - 50 years (1500\%). The number of PGs performed decreased overall, especially in patients aged $<60$ years, but it increased among patients $>80$ years with the highest percentage change noted in patients $>90$ years (13\%).

\subsection{Pathology and Co-Morbidities}

Gastric carcinoma was the most common indication for 
Table 1. Complications studied by ICD code and their broad grouping for analysis (national inpatient sample database 2004-2008).

\begin{tabular}{|c|c|c|c|}
\hline Broad group & ICD 9 code & Complication & Components \\
\hline \multicolumn{4}{|l|}{$\begin{array}{l}\text { Medical } \\
\text { complications }\end{array}$} \\
\hline $\begin{array}{l}\text { Respiratory } \\
\text { complications }\end{array}$ & $\begin{array}{c}512.1 \\
415.11 \\
518.5 \\
518.4 \\
997.31 \\
997.39\end{array}$ & $\begin{array}{l}\text { Iatrogenic pneumothorax } \\
\text { Iatrogenic pulmonary embolic infarct } \\
\text { Post traumatic pulmonary insufficiency } \\
\text { Acute lung edema NOS } \\
\text { Ventilator associated pneumonia } \\
\text { Respiratory complications NEC }\end{array}$ & \\
\hline $\begin{array}{l}\text { Cardiac } \\
\text { complications }\end{array}$ & 997.1 & Post-operative complications of Heart & $\begin{array}{l}\text { Cardiac arrest during or resulting from a procedure } \\
\text { Cardiac insufficiency during or resulting from a procedure } \\
\text { Cardiorespiratory failure during or resulting from a procedure } \\
\text { Heart failure during or resulting from a procedure }\end{array}$ \\
\hline $\begin{array}{l}\text { Urinary tract } \\
\text { complications }\end{array}$ & 997.5 & $\begin{array}{l}\text { Post-operative complications of } \\
\text { urinary tract }\end{array}$ & $\begin{array}{l}\text { Complications of: } \\
\text { External stoma of urinary tract } \\
\text { Internal anastomosis and bypass of urinary tract, including that } \\
\text { involving intestinal tract } \\
\text { Oliguria or anuria specified as due to procedure } \\
\text { Renal failure (acute) specified as due to procedure } \\
\text { Insufficiency (acute) specified as due to procedure } \\
\text { Tubular necrosis (acute) specified as due to procedure }\end{array}$ \\
\hline Sepsis & 995.91 & & $\begin{array}{l}\text { Intra-abdominal post-operative } \\
\text { Stitch post-operative } \\
\text { Subphrenic post-operative } \\
\text { Wound post-operative }\end{array}$ \\
\hline \multicolumn{4}{|l|}{$\begin{array}{l}\text { Surgical } \\
\text { complications }\end{array}$} \\
\hline Fistula of bile duct & 576.4 & Fistula of bile duct & \\
\hline $\begin{array}{l}\text { Post-operative } \\
\text { infection }\end{array}$ & 998.59 & Other post-operative infection & Abscess: post-operative \\
\hline $\begin{array}{l}\text { Accidental operative } \\
\text { laceration }\end{array}$ & 998.2 & Accidental operative laceration & $\begin{array}{l}\text { Accidental perforation by catheter or other instrument during a } \\
\text { procedure on: } \\
\text { Blood vessel, Nerve, Organ }\end{array}$ \\
\hline $\begin{array}{l}\text { Post-operative } \\
\text { hemorrhage }\end{array}$ & 998.11 & Post-operative hemorrhage & \\
\hline $\begin{array}{l}\text { Disruption of } \\
\text { internal operative } \\
\text { wound }\end{array}$ & 998.31 & Disruption of internal operative wound & \\
\hline $\begin{array}{l}\text { Disruption of } \\
\text { external operative } \\
\text { wound }\end{array}$ & 998.32 & Disruption of external operative wound & \\
\hline Intestinal fistula & 569.81 & Intestinal fistula & \\
\hline $\begin{array}{l}\text { Deep vein } \\
\text { thrombosis }\end{array}$ & $\begin{array}{c}453.9 \\
453.40 \\
453.41 \\
453.42\end{array}$ & $\begin{array}{l}\text { Venous thrombosis NOS } \\
\text { DVT/Embolism lower ext NOS } \\
\text { DVT/Embolism proximal lower ext } \\
\text { DVT/Embolism distal lower ext }\end{array}$ & \\
\hline
\end{tabular}




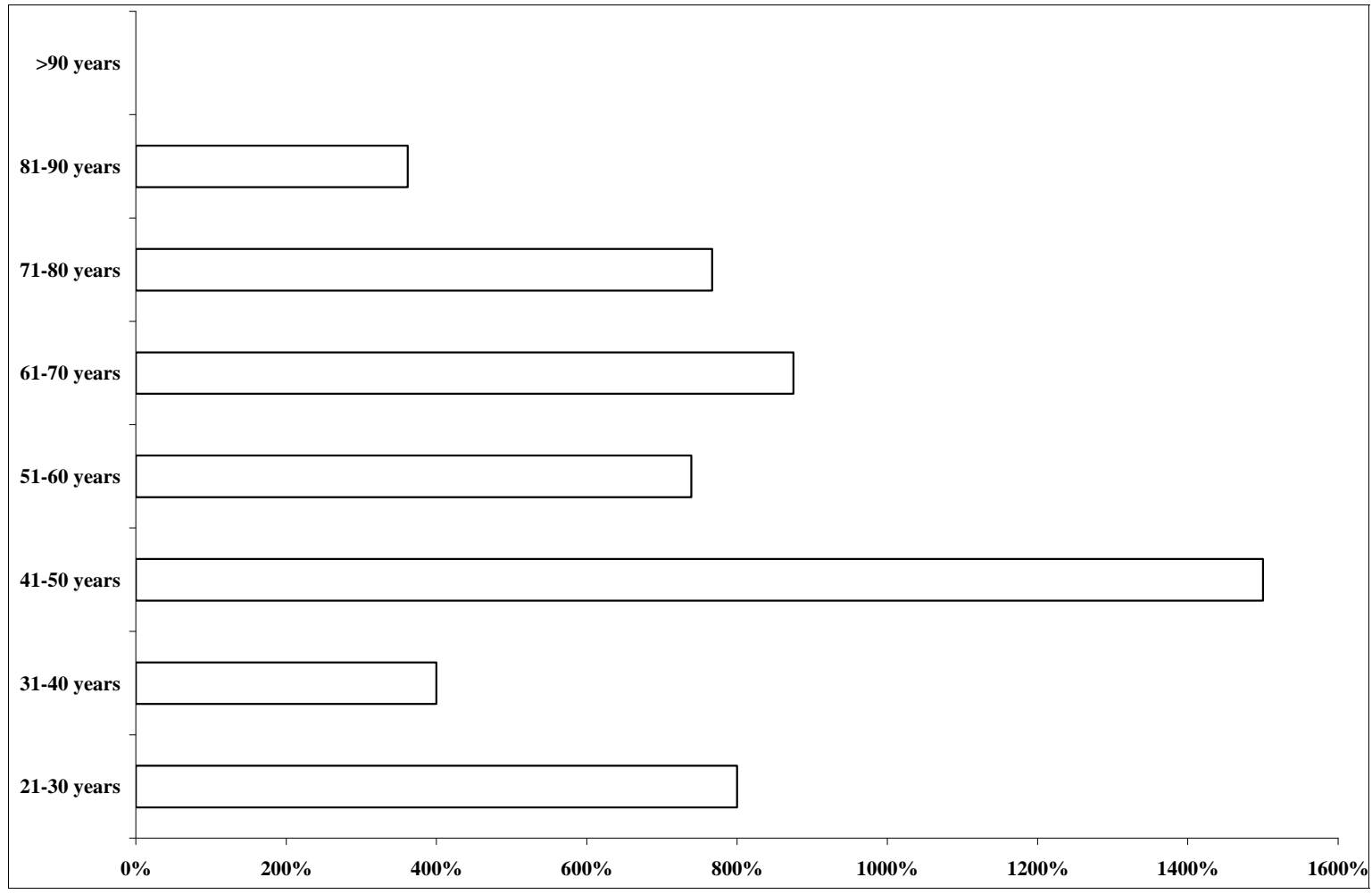

(a)

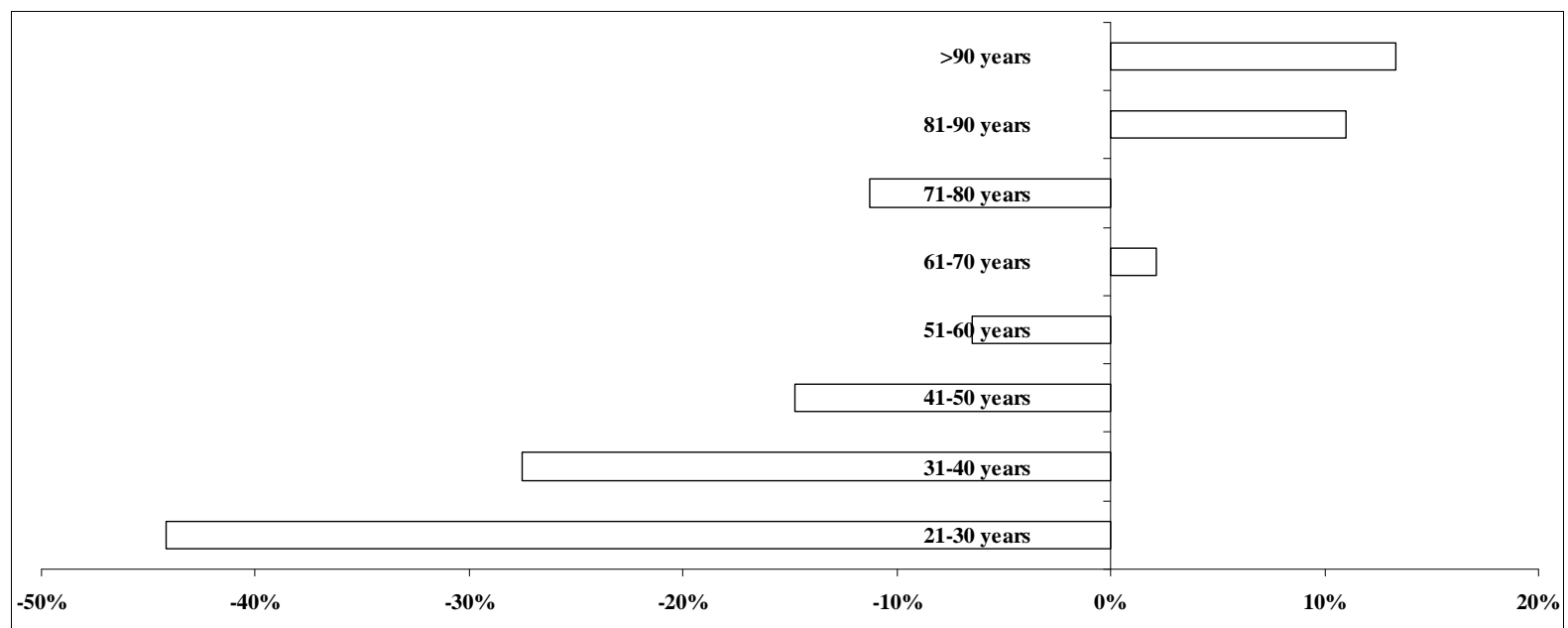

(b)

Figure 1. (a) Percentage change in the number of total gastrectomy performed among all age groups between 2004-2008 (national inpatient sample database); (b) percentage change in the number of partial gastrectomy among all age groups between 2004-2008 (national inpatient sample database).

TG overall (87.5\%) and in each age group. Benign disease was the most common indication for PG overall (57\%) and for all patients under 70 years. Gastric carcinoma was the most common indication for PG in patients $>70$ years (Table 3(a)). An increasing number of PGs were performed for both malignant and benign disease with advancing age, while an increasing percentage of TGs compared to PGs were performed in younger patients (pri- marily in those with malignant disease). (Table 3(a)) Over the course of the study, there was an increasing trend in the number of patients operated upon for gastric cancer, with a corresponding decrease in the number of patients operated upon for benign disease in all but the seventh and tenth decades (Table 3(b)). Gastroesophageal junction (GEJ) or abdominal esophagus carcinoma was the most indication for total gastrectomy in all age 
Table 2. Clinicopathological data on 13,799 patients undergoing total and partial gastrectomy (national inpatient sample database 2004-2008).

\begin{tabular}{|c|c|c|c|c|c|c|c|c|c|c|}
\hline & \multicolumn{10}{|c|}{ Patients' Age Groups by Decade } \\
\hline & Overall & $21-30$ & $31-40$ & $41-50$ & $51-60$ & $61-70$ & $71-80$ & $81-90$ & $>91$ & $\begin{array}{c}p \\
\text { value }\end{array}$ \\
\hline \multicolumn{11}{|l|}{ Total Gastrectomy } \\
\hline $\mathrm{N},(\%)$ & 3271 & $32(1.0)$ & $110(3.4)$ & 356 (10.9) & $733(22.4)$ & $980(30.0)$ & $802(24.5)$ & $250(7.6)$ & $(-)$ & \\
\hline Male: Female ratio & 2.1: 1 & 0.9: 1 & 1.2: 1 & 1.7: 1 & 2.2: 1 & 2.9: 1 & 2.1: 1 & 1.7: 1 & 1:01 & 0.001 \\
\hline Non-elective admission, N (\%) & $695(21.2)$ & $11(34.4)$ & $25(22.7)$ & 66 (18.5) & $152(20.7)$ & $174(17.8)$ & $168(20.9)$ & $94(37.6)$ & $(-)$ & 0.001 \\
\hline Pre-operative LOS, mean \pm SD & $1.2 \pm 3.8$ & $1.8 \pm 4.6$ & $0.7 \pm 2.6$ & $1.0 \pm 3.1$ & $1.0 \pm 2.8$ & $1.0 \pm 3.5$ & $1.5 \pm 5.0$ & $2.3 \pm 4.1$ & $6.4 \pm 5.6$ & 0.001 \\
\hline Total LOS, mean \pm SD & $16.5 \pm 14.7$ & $16.2 \pm 16.7$ & $13.5 \pm 9.3$ & $14.8 \pm 12.7$ & $15.5 \pm 13.2$ & $16.7 \pm 15.4$ & $17.7 \pm 15.4$ & $18.7 \pm 17.0$ & $17.4 \pm 8.5$ & 50.001 \\
\hline Mortality, N (\%) & $250(7.6)$ & $(-)$ & $(-)$ & $11(3.1)$ & $39(5.3)$ & $60(6.1)$ & $88(11.0)$ & 45 (18.0) & $(-)$ & 0.003 \\
\hline \multicolumn{11}{|l|}{ Partial Gastrectomy } \\
\hline $\mathrm{N},(\%)$ & 10,528 & $153(1.5)$ & $531(5.0)$ & $1336(12.7)$ & $2158(20.5)$ & 2349 & $2436(23.1)$ & 1429 & $136(1.3)$ & \\
\hline Male: Female ratio & $1: 1.1$ & $1: 1.6$ & $1: 1.5$ & $1: 1.3$ & 1:1.1 & 1:0.9 & $1: 1.0$ & $1: 1.3$ & $1: 1.4$ & 0.001 \\
\hline Non-elective admission, N (\%) & $4884(46.5)$ & 87 (56.9) & $246(46.4)$ & $567(42.5)$ & $932(43.4)$ & $1001(42.7)$ & $1142(46.9)$ & 820 & $89(65.4)$ & 0.001 \\
\hline Pre-operative LOS, mean \pm SD & $2.1 \pm 4.4$ & $2.6 \pm 5.1$ & $2.3 \pm 8.5$ & $1.7 \pm 3.6$ & $2.0 \pm 4.2$ & $1.9 \pm 3.9$ & $2.2 \pm 4.0$ & $2.9 \pm 4.4$ & $3.3 \pm 4.3$ & 0.001 \\
\hline Total LOS, mean \pm SD & $13.9 \pm 13.4$ & $13.7 \pm 18.3$ & $11.7 \pm 17.8$ & $12.2 \pm 11.9$ & $12.9 \pm 13.3$ & $13.9 \pm 13.2$ & $14.9 \pm 13.6$ & $16.1 \pm 12.2$ & $15.8 \pm 9.8$ & 30.001 \\
\hline Mortality, N (\%) & $670(6.4)$ & $(-)$ & $(-)$ & $31(2.3)$ & $61(2.8)$ & 135 (5.7) & 199 (8.2) & $206(14.4)$ & 27 (19.9) & 0.001 \\
\hline
\end{tabular}

Abbreviations: N: number of patients; LOS: length of stay; SD: standard deviation; (-): As per NIS database reporting guidelines, values <10 are not shown in the table; ${ }^{*} p$ value $<0.05$ statistical significance for the range.

Table 3. (a) Distribution of malignant and benign cases in 13,799 patients undergoing total and partial gastrectomy separated according to patient age groups by decades (national inpatient sample database 2004-2008); (b) Distribution of malignant and benign cases in 13,799 patients undergoing total and partial gastrectomy separated according to patient age groups by decades and year of study period (national inpatient sample database 2004-2008).

(a)

\begin{tabular}{|c|c|c|c|c|c|}
\hline \multirow{3}{*}{ Age groups } & \multicolumn{4}{|c|}{ Main Group } & \multirow{3}{*}{$p$ value } \\
\hline & \multicolumn{2}{|c|}{ Malignant disease, N (\%) } & \multicolumn{2}{|c|}{ Benign disease, $\mathrm{N}(\%)$} & \\
\hline & Total gastrectomy & Partial gastrectomy & Total gastrectomy & Partial gastrectomy & \\
\hline $21-30$ & $19(50.0)$ & $19(50.0)$ & $13(8.8)$ & $135(91.2)$ & 0.001 \\
\hline $31-40$ & 79 (40.7) & $115(59.3)$ & $31(6.9)$ & $420(93.1)$ & 0.001 \\
\hline $41-50$ & $280(43.1)$ & 370 (56.9) & $76(7.3)$ & $970(92.7)$ & 0.001 \\
\hline $51-60$ & $634(47.3)$ & 706 (52.7) & $99(6.3)$ & $1462(93.7)$ & 0.001 \\
\hline $61-70$ & $884(45.1)$ & 1074 (54.9) & $96(7.0)$ & $1284(93.0)$ & 0.001 \\
\hline $71-80$ & $736(35.0)$ & $1367(65.0)$ & $66(5.8)$ & $1075(94.2)$ & 0.001 \\
\hline $81-90$ & $222(20.7)$ & 849 (79.3) & $28(4.6)$ & $583(95.4)$ & 0.001 \\
\hline$>90$ & $(-)$ & $74(90.2)$ & $0(0)$ & 63 (100.0) & 0.001 \\
\hline
\end{tabular}

Abbreviations: N: number of patients; (-): As per NIS database reporting guidelines, values $<10$ are not shown in the table; ${ }^{*} p$ value $<0.05$ statistical significance for the range.

(b)

\begin{tabular}{ccccccccccc}
\hline \multirow{2}{*}{$\begin{array}{c}\text { Age } \\
\text { Groups }\end{array}$} & \multicolumn{9}{c}{ Number of Malignant and Benign cases during study period (2004-2008) } \\
\cline { 2 - 12 }$y$ & \multicolumn{7}{c}{ Malignant disease, N (\%) } & \multicolumn{5}{c}{ Benign disease, N (\%) } \\
\hline $21-30$ & $(-)$ & $12(31.6)$ & $(-)$ & $7(18.4)$ & $8(21.1)$ & $43(29.1)$ & $28(18.9)$ & $19(12.8)$ & $32(21.6)$ & $26(17.6)$ \\
$31-40$ & $26(13.4)$ & $40(20.6)$ & $41(21.1)$ & $47(24.2)$ & $40(20.6)$ & $130(28.8)$ & $86(19.1)$ & $74(16.4)$ & $68(15.1)$ & $93(20.6)$ \\
$41-50$ & $76(11.7)$ & $143(22.0)$ & $101(15.5)$ & $162(24.9)$ & $168(25.8)$ & $242(23.1)$ & $215(20.6)$ & $195(18.6)$ & $184(17.6)$ & $210(20.1)$ \\
$51-60$ & $154(11.5)$ & $283(21.1)$ & $268(20.0)$ & $331(24.7)$ & $304(22.7)$ & $344(22.0)$ & $295(18.9)$ & $320(20.5)$ & $268(17.2)$ & $334(21.4)$ \\
$61-70$ & $237(12.1)$ & $397(20.3)$ & $433(22.1)$ & $432(22.1)$ & $459(23.4)$ & $267(19.3)$ & $286(20.7)$ & $269(19.5)$ & $256(18.6)$ & $302(21.9)$ \\
$71-80$ & $279(13.3)$ & $474(22.5)$ & $422(20.1)$ & $468(22.3)$ & $460(21.9)$ & $258(22.6)$ & $240(21.0)$ & $208(18.2)$ & $231(20.2)$ & $204(17.9)$ \\
$81-90$ & $158(14.8)$ & $230(21.5)$ & $210(19.6)$ & $219(20.4)$ & $254(23.7)$ & $129(21.1)$ & $140(22.9)$ & $129(21.1)$ & $103(16.9)$ & $110(18.0)$ \\
$>90$ & $17(20.7)$ & $12(14.6)$ & $18(22.0)$ & $13(15.9)$ & $22(26.8)$ & $13(20.6)$ & $(-)$ & $14(22.2)$ & $11(17.5)$ & $15(23.8)$ \\
\hline
\end{tabular}

Abbreviations: N: number of patients; (-): As per NIS database reporting guidelines, values $<10$ are not shown in the table. 
groups (59.2\%), whereas partial gastrectomy for malignnancy was most commonly performed for antral or pyloric tumors (47.2\%) in all age groups (Table 4). Overall, $15.1 \%$ of gastrectomies were performed for GEJ/abdominal esophagus tumors, while $17.5 \%$ were performed for antral tumors. Elective admissions were far more common in the TG group ( $\mathrm{N}=2576,78.8 \%)$ compared to the PG group ( $\mathrm{N}=5648,53.6 \%$ ) with more non-elective admissions occurring in the both the youngest and oldest age groups (Table 2). The three most common co-morbidities overall were hypertension (TG; $43.7 \%$ vs. PG; 45.5\%), fluid and electrolyte imbalances (TG; 27\%) vs. PG; 28.1\%), and chronic pulmonary disease (TG; $9.9 \%$ vs. PG; $18.2 \%$ ). (Tables 5(a) and (b)) The percentage of patients with hypertension and fluid and electrolyte imbalances was highest in those $>80$ years for both the TG and PG groups. Chronic pulmonary disease occurred most commonly in those aged 71 - 80 years for both the TG and PG groups, but occurred with high frequency in all patients $>70$ years $(\sim 19 \%-26 \%)$.

\subsection{Complications}

Complications in the TG and PG groups are detailed in Tables 6(a) and (b). The overall complication rate was $54 \%$ in the TG group and 36\% in the PG group. The most common complications in the TG group were respiratory (18\%) and gastrointestinal (GI) (11.4\%), with the highest percent of complications occurring in those $>51$ years. An increasing number of respiratory complications were seen with advancing age, with those age 21 30 years having those lowest incidence $(9.4 \%)$ and those $>91$ having the highest (25\%). The age-related trend in GI complications (intestinal obstruction and anastomotic and hepatic complications) noted. Among PG patients, GI complications (9.6\%) and bile duct fistulas (7.2\%) were most common peaking incidence among those 61 70 years. Similar to the TG group, no appre- ciable age-related trend in GI complications was noted in the PG group. There was an increase age-related incidence in bile duct fistulas in the PG group ranging from $2 \%$ in those 21 - 30 years to $13.2 \%$ in those $>91$ years. Cardiac events occurred more commonly in the TG groups with the highest incidence in those $61-70(7.7 \%)$ and $71-80$ years old $(8.1 \%)(p<0.001)$. The overall cardiac complication rate in the PG group (3.2\%) was half of that observed in the TG group $(6.1 \%)(p<0.001)$.

\subsection{Clinicopathological Data and Mortality}

Clinical outcomes from the two surgery groups are detailed in Table 2. 59.5\% of all admissions for gastric surgery were elective, including $78.8 \%$ of TGs and $53.6 \%$ of PGs $(p=0.001)$. Elective admission status did not different for the PG groups, but was highest in those $>81$ years old. Elective admission status was far more common in those $<80$ years old undergoing TG compared to those $>81$ years old $(p=0.001)$. The mean pre-operative and total hospital LOS generally increased with advancing age for both the TG and PG with the exception of the youngest age group (age 21 - 30 years) whose LOS equaled those $>61$ years $(p<0.001)$. The overall mortality rate for all gastrectomy patients in the study groups was $6.7 \%$ (Table 7). The mortality rate for the TG group was $7.6 \%$, while the mortality rate for the PG group was 6.4\% $(p=0.01)$ (Table 2). Mortality rates increased in a linear fashion with advancing age also for both TG and PG beginning with those $>30$ years of age (Table 2). However, it is notable that the youngest group had a mortality rate higher than (TG group) or similar to (PG group) those aged 51 - 60 years $(p<0.001)$ (Table 7). The highest mortality rate of $20 \%$ was

Table 4. Anatomic site of malignancy in 13,799 patients undergoing total and partial gastrectomyseparated according to patient age groups by decades (national inpatient sample database 2004-2008).

\begin{tabular}{|c|c|c|c|c|c|c|c|c|c|c|c|c|c|c|c|c|c|c|}
\hline & \multicolumn{9}{|c|}{ Total Gastrectomy, N (\%) } & \multicolumn{9}{|c|}{ Partial Gastrectomy, N (\%) } \\
\hline $\begin{array}{c}\text { Site of } \\
\text { Malignancy }\end{array}$ & Overall & $21-30$ & $31-40$ & $41-50$ & $51-60$ & $61-70$ & $71-80$ & $81-90$ & $>91$ & Overall & $21-30$ & $31-40$ & $41-50$ & $51-60$ & $61-70$ & $71-80$ & $81-90$ & $>91$ \\
\hline $\begin{array}{l}\text { Lower } 1 / 3^{\text {rd }} \\
\text { and } \\
\text { Abdominal } \\
\text { Esophagus }\end{array}$ & $\begin{array}{c}422 \\
(15.6)\end{array}$ & 0 & $\begin{array}{c}11 \\
(13.6)\end{array}$ & $\begin{array}{c}41 \\
(16)\end{array}$ & $\begin{array}{c}100 \\
(16.8)\end{array}$ & $\begin{array}{c}150 \\
(18.2)\end{array}$ & $\begin{array}{c}94 \\
(13.7)\end{array}$ & $\begin{array}{c}26 \\
(11.7)\end{array}$ & 0 & $\begin{array}{c}59 \\
(1.3)\end{array}$ & 0 & $(-)$ & $(-)$ & $\begin{array}{c}18 \\
(2.5)\end{array}$ & $\begin{array}{c}22 \\
(2.1)\end{array}$ & $(-)$ & $(-)$ & 0 \\
\hline $\begin{array}{c}\text { GE } \\
\text { Junction }\end{array}$ & $\begin{array}{c}1176 \\
(43.6)\end{array}$ & $(-)$ & $\begin{array}{c}30 \\
(37)\end{array}$ & $\begin{array}{c}106 \\
(41.4)\end{array}$ & $\begin{array}{c}279 \\
(46.9)\end{array}$ & $\begin{array}{c}388 \\
(47.1)\end{array}$ & $\begin{array}{c}296 \\
(43.1)\end{array}$ & $\begin{array}{c}70 \\
(31.5)\end{array}$ & $(-)$ & $\begin{array}{c}428 \\
(9.4)\end{array}$ & $(-)$ & $(-)$ & $\begin{array}{c}41 \\
(11.2)\end{array}$ & $\begin{array}{c}69 \\
(9.8)\end{array}$ & $\begin{array}{c}99 \\
(9.2)\end{array}$ & $\begin{array}{c}143 \\
(10.4)\end{array}$ & $\begin{array}{c}63 \\
(7.4)\end{array}$ & $(-)$ \\
\hline $\begin{array}{l}\text { Gastric } \\
\text { Body }\end{array}$ & $\begin{array}{c}167 \\
(6.2)\end{array}$ & $(-)$ & $(-)$ & $\begin{array}{c}18 \\
(7.0)\end{array}$ & $\begin{array}{c}21 \\
(3.5)\end{array}$ & $\begin{array}{c}40 \\
(4.9)\end{array}$ & $\begin{array}{c}59 \\
(8.6)\end{array}$ & $\begin{array}{l}20 \\
(9)\end{array}$ & $(-)$ & $\begin{array}{c}483 \\
(10.6)\end{array}$ & $(-)$ & $\begin{array}{c}15 \\
(13.3)\end{array}$ & $\begin{array}{c}38 \\
(10.4)\end{array}$ & $\begin{array}{c}76 \\
(10.7)\end{array}$ & $\begin{array}{c}101 \\
(9.4)\end{array}$ & $\begin{array}{c}168 \\
(12.2)\end{array}$ & $\begin{array}{c}73 \\
(8.6)\end{array}$ & $(-)$ \\
\hline $\begin{array}{l}\text { Antrum/ } \\
\text { Pyloric/ } \\
\text { Pre-pyloric } \\
\text { Tumor }\end{array}$ & $\begin{array}{c}261 \\
(9.7)\end{array}$ & $(-)$ & $(-)$ & $\begin{array}{c}28 \\
(10.9)\end{array}$ & $\begin{array}{c}64 \\
(10.8)\end{array}$ & $\begin{array}{c}75 \\
(9.1)\end{array}$ & $\begin{array}{c}53 \\
(7.7)\end{array}$ & $\begin{array}{c}27 \\
(12.2)\end{array}$ & $(-)$ & $\begin{array}{c}2159 \\
(47.2)\end{array}$ & $(-)$ & $\begin{array}{c}53 \\
(46.9)\end{array}$ & $\begin{array}{c}158 \\
(43.3)\end{array}$ & $\begin{array}{c}299 \\
(42.3)\end{array}$ & $\begin{array}{c}526 \\
(49.1)\end{array}$ & $\begin{array}{c}662 \\
(48.1)\end{array}$ & $\begin{array}{c}414 \\
(48.7)\end{array}$ & $\begin{array}{c}41 \\
(55.4)\end{array}$ \\
\hline
\end{tabular}

Abbreviations: N: number of patients; GE: gastroesophageal; NEC: not elsewhere classified; NOS: not otherwise specified; (-): As per NIS database reporting guidelines, values $<10$ are not shown in the table. 
Table 5. (a) Distribution of co-morbidities in 3271 patients undergoing total gastrectomy separated according to patient age groups by decades (national inpatient sample database 2004-2008); (b) distribution of co-morbidities in 10,528 patients undergoing partial gastrectomy separated according to patient age groups by decades (national inpatient sample database 2004-2008).

(a)

\begin{tabular}{|c|c|c|c|c|c|c|c|c|c|c|}
\hline \multirow{2}{*}{ Co-morbidities } & \multirow{2}{*}{ Overall } & \multicolumn{8}{|c|}{ Patients’ Age Groups by Decades, N (\%) } & \multirow{2}{*}{$p$ value ${ }^{*}$} \\
\hline & & $21-30$ & $31-40$ & $41-50$ & $51-60$ & $61-70$ & $71-80$ & $81-90$ & $>91$ & \\
\hline Hypertension & 1429 (43.7) & $(-)$ & $(-)$ & $\begin{array}{c}72 \\
(20.2)\end{array}$ & $\begin{array}{c}291 \\
(39.7)\end{array}$ & $\begin{array}{c}473 \\
(48.3)\end{array}$ & $\begin{array}{c}438 \\
(54.6)\end{array}$ & $\begin{array}{c}138 \\
(55.2)\end{array}$ & $(-)$ & 0.001 \\
\hline $\begin{array}{l}\text { Fluid and electrolyte } \\
\text { imbalances }\end{array}$ & $882(27.0)$ & $(-)$ & $\begin{array}{c}27 \\
(24.5)\end{array}$ & $\begin{array}{c}74 \\
(20.8)\end{array}$ & $\begin{array}{c}173 \\
(23.6)\end{array}$ & $\begin{array}{c}284 \\
(29.0)\end{array}$ & $\begin{array}{c}224 \\
(27.9)\end{array}$ & $87(34.8)$ & $(-)$ & 0.001 \\
\hline $\begin{array}{l}\text { Chronic pulmonary } \\
\text { disease }\end{array}$ & 651 (19.9) & $(-)$ & $(-)$ & $\begin{array}{c}52 \\
(14.6)\end{array}$ & $\begin{array}{c}114 \\
(15.6)\end{array}$ & $\begin{array}{c}211 \\
(21.5)\end{array}$ & $\begin{array}{c}212 \\
(26.4)\end{array}$ & $48(19.2)$ & $(-)$ & 0.001 \\
\hline Weight loss & $480(14.7)$ & $(-)$ & $\begin{array}{c}15 \\
(13.6)\end{array}$ & $\begin{array}{c}48 \\
(13.5)\end{array}$ & $\begin{array}{c}92 \\
(12.6)\end{array}$ & $\begin{array}{c}134 \\
(13.7)\end{array}$ & $\begin{array}{c}132 \\
(16.5)\end{array}$ & $53(21.2)$ & $(-)$ & 0.043 \\
\hline Obesity & $186(5.7)$ & 0 & $(-)$ & $26(7.3)$ & $65(8.9)$ & $58(5.9)$ & $28(3.5)$ & $5(2.0)$ & 0 & 0.001 \\
\hline Coagulopathy & $143(4.4)$ & $(-)$ & $(-)$ & $10(2.8)$ & $25(3.4)$ & $41(4.2)$ & $44(5.5)$ & $19(7.6)$ & $(-)$ & 0.017 \\
\hline $\begin{array}{l}\text { Chronic blood loss } \\
\text { anemia }\end{array}$ & $143(4.4)$ & $(-)$ & $(-)$ & $(-)$ & $30(4.1)$ & 34 (3.5) & $52(6.5)$ & $18(7.2)$ & 0 & 0.001 \\
\hline Renal failure & 118 (3.6) & $(-)$ & $(-)$ & $(-)$ & $19(2.6)$ & 33 (3.4) & $41(5.1)$ & $18(7.2)$ & 0 & 0.001 \\
\hline Alcohol abuse & $104(3.2)$ & $(-)$ & $(-)$ & $16(4.5)$ & $37(5.0)$ & $31(3.2)$ & $17(2.1)$ & 0 & 0 & 0.002 \\
\hline Liver disease & $75(2.3)$ & 0 & $(-)$ & $11(3.1)$ & $17(2.3)$ & $18(1.8)$ & $22(2.7)$ & $(-)$ & 0 & 0.76 \\
\hline Diabetes & $47(1.4)$ & $(-)$ & $(-)$ & $(-)$ & $15(2.0)$ & $13(1.3)$ & $12(1.5)$ & $(-)$ & $(-)$ & 0.83 \\
\hline
\end{tabular}

Abbreviations: N: number of patients; (-): As per NIS database reporting guidelines, values $<10$ are not shown in the table; ${ }^{*} p$ value $<0.05$ statistical significance for the range.

(b)

\begin{tabular}{|c|c|c|c|c|c|c|c|c|c|c|}
\hline \multirow{2}{*}{ Co-morbidities } & \multirow{2}{*}{ Overall } & \multicolumn{8}{|c|}{ Patients’ Age Groups by Decades, N (\%) } & \multirow{2}{*}{$p$ value* } \\
\hline & & $21-30$ & $31-40$ & $41-50$ & $51-60$ & $61-70$ & $71-80$ & $81-90$ & $>91$ & \\
\hline Hypertension & $4875(45.5)$ & $\begin{array}{c}24 \\
(15.6)\end{array}$ & $\begin{array}{l}103 \\
(19.1)\end{array}$ & $\begin{array}{c}344 \\
(25.2)\end{array}$ & $\begin{array}{c}888 \\
(40.5)\end{array}$ & $\begin{array}{l}1224 \\
(51.0)\end{array}$ & $\begin{array}{l}1415 \\
(57.2)\end{array}$ & $\begin{array}{c}792 \\
(54.9)\end{array}$ & $\begin{array}{c}85 \\
(62.0)\end{array}$ & 0.001 \\
\hline $\begin{array}{l}\text { Fluid and electrolyte } \\
\text { imbalances }\end{array}$ & $3011(28.1)$ & $\begin{array}{c}31 \\
(20.1)\end{array}$ & $\begin{array}{c}106 \\
(19.7)\end{array}$ & $\begin{array}{c}302 \\
(22.1)\end{array}$ & $\begin{array}{c}536 \\
(24.4)\end{array}$ & $\begin{array}{c}642 \\
(26.8)\end{array}$ & $\begin{array}{c}789 \\
(31.9)\end{array}$ & $\begin{array}{c}539 \\
(37.4)\end{array}$ & $\begin{array}{c}66 \\
(48.2)\end{array}$ & 0.001 \\
\hline $\begin{array}{l}\text { Chronic pulmonary } \\
\text { disease }\end{array}$ & 1949 (18.2) & $\begin{array}{c}12 \\
(7.8)\end{array}$ & $\begin{array}{c}55 \\
(10.2)\end{array}$ & $\begin{array}{c}184 \\
(13.5)\end{array}$ & $\begin{array}{c}364 \\
(16.6)\end{array}$ & $\begin{array}{c}487 \\
(20.3)\end{array}$ & $\begin{array}{c}549 \\
(22.2)\end{array}$ & $\begin{array}{c}270 \\
(18.7)\end{array}$ & $\begin{array}{c}28 \\
(20.4)\end{array}$ & 0.001 \\
\hline Weight loss & $1430(13.4)$ & $\begin{array}{c}19 \\
(12.3)\end{array}$ & $\begin{array}{c}67 \\
(12.4)\end{array}$ & $\begin{array}{c}163 \\
(11.9)\end{array}$ & $\begin{array}{c}243 \\
(11.1)\end{array}$ & $\begin{array}{c}307 \\
(12.8)\end{array}$ & $\begin{array}{c}352 \\
(14.2)\end{array}$ & $\begin{array}{l}257 \\
(17.8)\end{array}$ & $\begin{array}{c}22 \\
(16.1)\end{array}$ & 0.001 \\
\hline $\begin{array}{l}\text { Chronic blood loss } \\
\text { anemia }\end{array}$ & $1016(9.5)$ & $\begin{array}{c}12 \\
(7.8)\end{array}$ & $\begin{array}{c}47 \\
(8.7)\end{array}$ & $99(7.3)$ & $\begin{array}{l}175 \\
(8.0)\end{array}$ & $223(9.3)$ & $\begin{array}{c}259 \\
(10.5)\end{array}$ & $\begin{array}{c}186 \\
(12.9)\end{array}$ & $\begin{array}{c}15 \\
(10.9)\end{array}$ & 0.001 \\
\hline Obesity & $548(5.1)$ & $(-)$ & $\begin{array}{c}43 \\
(8.0)\end{array}$ & $\begin{array}{l}102 \\
(7.5)\end{array}$ & $\begin{array}{l}159 \\
(7.2)\end{array}$ & $131(5.5)$ & $71(2.9)$ & $30(2.1)$ & $(-)$ & 0.001 \\
\hline Renal failure & $543(5.1)$ & $(-)$ & $\begin{array}{c}12 \\
(2.2)\end{array}$ & $37(2.7)$ & $63(2.9)$ & $107(4.5)$ & $\begin{array}{l}173 \\
(7.0)\end{array}$ & $130(9.0)$ & $\begin{array}{c}16 \\
(11.7)\end{array}$ & 0.001 \\
\hline Coagulopathy & $471(4.4)$ & $(-)$ & $\begin{array}{c}13 \\
(2.4)\end{array}$ & $41(3.0)$ & $79(3.6)$ & $95(4.0)$ & $\begin{array}{c}139 \\
(5.6)\end{array}$ & $89(6.2)$ & $(-)$ & 0.001 \\
\hline Liver disease & $396(3.7)$ & $(-)$ & $\begin{array}{c}32 \\
(5.9)\end{array}$ & $65(4.8)$ & $\begin{array}{l}115 \\
(5.2)\end{array}$ & 87 (3.6) & $69(2.8)$ & $19(1.3)$ & $(-)$ & 0.001 \\
\hline Alcohol abuse & 379 (3.5) & $(-)$ & $\begin{array}{c}24 \\
(4.5)\end{array}$ & $93(6.8)$ & $\begin{array}{l}102 \\
(4.6)\end{array}$ & $89(3.7)$ & $57(2.3)$ & $(-)$ & $(-)$ & 0.001 \\
\hline Diabetes & 197 (1.8) & $(-)$ & $(-)$ & $(-)$ & 38 (1.7) & $59(2.5)$ & $59(2.4)$ & $26(1.8)$ & $(-)$ & 0.001 \\
\hline
\end{tabular}

Abbreviations: N: number of patients; (-): As per NIS database reporting guidelines, values $<10$ are not shown in the table; ${ }^{*} p$ value $<0.05$ statistical significance for the range. 
Table 6. (a) Distribution of post-operative complications in 3271 patients undergoing total gastrectomy separated according to patient age groups by decades (national inpatient sample database 2004-2008); (b) Distribution of post-operative complications in 10,528 patients undergoing partial gastrectomy separated according to patient age groups by decades (national inpatient sample database 2004-2008).

(a)

\begin{tabular}{|c|c|c|c|c|c|c|c|c|c|}
\hline \multirow[b]{2}{*}{ Complications } & \multirow[b]{2}{*}{ Overall } & \multicolumn{8}{|c|}{ Patients’ Age Groups by Decade, N (\%) } \\
\hline & & $21-30$ & $31-40$ & $41-50$ & $51-60$ & $61-70$ & $71-80$ & $81-90$ & $>91$ \\
\hline \multicolumn{10}{|l|}{ Medical Complications } \\
\hline Respiratory complications & $590(18.0)$ & $(-)$ & $12(10.9)$ & $44(12.4)$ & $117(16.0)$ & $207(21.1)$ & 158 (19.7) & $47(18.8)$ & $(-)$ \\
\hline Cardiac complications & $201(6.1)$ & $(-)$ & $(-)$ & $15(4.2)$ & $29(4.0)$ & $75(7.7)$ & $65(8.1)$ & $12(4.8)$ & 0 \\
\hline Urinary complications & $47(1.4)$ & 0 & $1(0.9)$ & $(-)$ & $(-)$ & $13(1.3)$ & $16(2.0)$ & $(-)$ & $(-)$ \\
\hline Post-operative stroke & $5(0.2)$ & 0 & 0 & $(-)$ & 0 & $(-)$ & $(-)$ & 0 & 0 \\
\hline Sepsis & $3(0.09)$ & 0 & 0 & 0 & 0 & $(-)$ & 0 & 0 & 0 \\
\hline \multicolumn{10}{|l|}{ Surgical Complications } \\
\hline Gastrointestinal complications & $374(11.4)$ & $(-)$ & $12(10.9)$ & $42(11.8)$ & $85(11.6)$ & $120(12.2)$ & 85 (10.6) & $28(11.2)$ & $(-)$ \\
\hline Other post-operative infection & $208(6.4)$ & $(-)$ & $(-)$ & $25(7.0)$ & $45(6.1)$ & $74(7.6)$ & $44(5.5)$ & $13(5.2)$ & 0 \\
\hline Accidental laceration during a procedure & $112(3.4)$ & 0 & $(-)$ & $14(3.9)$ & $29(4.0)$ & $36(3.7)$ & $19(2.4)$ & $9(3.6)$ & $(-)$ \\
\hline Post-operative Hemorrhage & $83(2.5)$ & $(-)$ & $(-)$ & $12(3.4)$ & $17(2.3)$ & $19(1.9)$ & $23(2.9)$ & $9(3.6)$ & 0 \\
\hline Venous thrombosis & $53(1.6)$ & 0 & $(-)$ & $(-)$ & $(-)$ & $20(2.0)$ & $15(1.9)$ & $(-)$ & 0 \\
\hline Disruption of external operation wound & $39(1.2)$ & 0 & $(-)$ & $(-)$ & $(-)$ & $20(2.0)$ & - & 0 & 0 \\
\hline Disruption of internal operation wound & $35(1.1)$ & 0 & 0 & $(-)$ & $(-)$ & $(-)$ & $12(1.5)$ & $(-)$ & 0 \\
\hline Intestinal fistula & $3(0.1)$ & 0 & 0 & 0 & $(-)$ & 0 & - & 0 & 0 \\
\hline
\end{tabular}

Abbreviations: N: number of patients; (-): As per NIS database reporting guidelines, values $<10$ are not shown in the table.

(b)

\begin{tabular}{|c|c|c|c|c|c|c|c|c|c|}
\hline \multirow[b]{2}{*}{ Complications } & \multirow[b]{2}{*}{ Overall } & \multicolumn{8}{|c|}{ Patients’ Age Groups by Decades, N (\%) } \\
\hline & & $21-30$ & $31-40$ & $41-50$ & $51-60$ & $61-70$ & $71-80$ & $81-90$ & $>91$ \\
\hline \multicolumn{10}{|l|}{ Medical Complications } \\
\hline Cardiac Complications & $333(3.2)$ & $(-)$ & $(-)$ & $15(1.1)$ & $40(1.9)$ & $61(2.6)$ & $105(4.3)$ & $91(6.4)$ & $12(8.8)$ \\
\hline Respiratory Complications & $160(1.5)$ & $(-)$ & $(-)$ & $21(1.6)$ & $27(1.3)$ & $46(2)$ & $31(1.3)$ & $24(1.7)$ & $(-)$ \\
\hline Urinary Tract Complications & $97(0.9)$ & 0 & $(-)$ & $(-)$ & $18(0.8)$ & $23(1.0)$ & $27(1.1)$ & $20(1.4)$ & $(-)$ \\
\hline Post-operative Stroke & $22(0.21)$ & 0 & 0 & $(-)$ & $(-)$ & $(-)$ & $(-)$ & $(-)$ & 0 \\
\hline Sepsis & $18(0.17)$ & 0 & 0 & 0 & $(-)$ & $(-)$ & $(-)$ & $(-)$ & 0 \\
\hline \multicolumn{10}{|l|}{ Surgical Complications } \\
\hline Gastrointestinal Complications & $1013(9.6)$ & $10(6.5)$ & $44(8.3)$ & $128(9.6)$ & $199(9.2)$ & $230(9.8)$ & $234(9.6)$ & $158(11.1)$ & $10(7.4)$ \\
\hline Fistula of bile duct & $763(7.2)$ & $(-)$ & $19(3.6)$ & $61(4.6)$ & $99(4.6)$ & $190(8.1)$ & $209(8.6)$ & $164(11.5)$ & $18(13.2)$ \\
\hline Post-operative infections & $568(5.4)$ & $10(6.5)$ & $21(4.0)$ & $71(5.3)$ & $123(5.7)$ & $137(5.8)$ & $119(4.9)$ & $76(5.3)$ & $11(8.1)$ \\
\hline Accidental operative laceration & $266(2.5)$ & 0 & $15(2.8)$ & $31(2.3)$ & $50(2.3)$ & $63(2.7)$ & $67(2.8)$ & $34(2.4)$ & $(-)$ \\
\hline Post-operative hemorrhage & $220(2.1)$ & $(-)$ & $12(2.3)$ & $26(1.9)$ & $35(1.6)$ & $61(2.6)$ & $47(1.9)$ & $34(2.4)$ & $(-)$ \\
\hline Disruption of external operation wound & $103(1.0)$ & $(-)$ & $(-)$ & $11(0.8)$ & $23(1.1)$ & $24(1.0)$ & $22(0.9)$ & $15(1.0)$ & $(-)$ \\
\hline Disruption of internal operation wound & $69(0.7)$ & $(-)$ & $(-)$ & $11(0.8)$ & $18(0.8)$ & $18(0.8)$ & $12(0.5)$ & $(-)$ & $(-)$ \\
\hline Intestinal fistula & $29(0.28)$ & 0 & $(-)$ & $(-)$ & $(-)$ & $(-)$ & $(-)$ & $(-)$ & 0 \\
\hline Deep vein thrombosis & $98(0.9)$ & 0 & $(-)$ & $(-)$ & $11(0.5)$ & $20(0.9)$ & $35(1.4)$ & $23(1.6)$ & $(-)$ \\
\hline
\end{tabular}

Abbreviations: N: number of patients; (-): As per NIS database reporting guidelines, values $<10$ are not shown in the table. 
Table 7. Univariate analysis of in-hospital mortality for 13,799 patients undergoing total and partial gastrectomy (national inpatient sample database 2004-2008).

\begin{tabular}{|c|c|c|}
\hline Variable & $\begin{array}{l}\text { Mortality } \\
\text { rate, } \%\end{array}$ & $p$ value ${ }^{*}$ \\
\hline Overall & 6.7 & \\
\hline Age groups & & 0.001 \\
\hline 21 - 30 years & 3.8 & \\
\hline 31 - 40 years & 1.4 & \\
\hline 41 - 50 years & 2.5 & \\
\hline 51 - 60 years & 3.4 & \\
\hline 61 - 70 years & 5.9 & \\
\hline 71 - 80 years & 8.9 & \\
\hline 81 - 90 years & 15.0 & \\
\hline$>90$ years & 20.0 & \\
\hline Gender & & 0.002 \\
\hline Male & 7.3 & \\
\hline Female & 6.0 & \\
\hline Race & & 0.015 \\
\hline Native American & 10.2 & \\
\hline White & 7.3 & \\
\hline Hispanic & 6.3 & \\
\hline Black & 5.7 & \\
\hline Asian or Pacific Islander & 5.1 & \\
\hline Others & 3.7 & \\
\hline Type of gastrectomy & & 0.011 \\
\hline Total gastrectomy & 7.6 & \\
\hline Partial gastrectomy & 6.4 & \\
\hline Indications for gastrectomy & & 0.001 \\
\hline Benign disease & 8.0 & \\
\hline Malignant disease & 5.6 & \\
\hline Type of admission & & 0.001 \\
\hline Non-elective admission & 11.4 & \\
\hline Elective admission & 3.5 & \\
\hline \multicolumn{3}{|l|}{ Co-morbidities } \\
\hline Coagulopathy & 25.2 & 0.001 \\
\hline Renal failure & 19.3 & 0.001 \\
\hline Congestive heart failure & 19.1 & 0.001 \\
\hline Fluid and electrolyte disorders & 13.0 & 0.001 \\
\hline Liver disease & 12.4 & 0.001 \\
\hline Peripheral vascular disorders & 12.0 & 0.001 \\
\hline Weight loss & 11.6 & 0.001 \\
\hline Diabetes with chronic complications & 9.9 & 0.042 \\
\hline Alcohol abuse & 9.9 & 0.005 \\
\hline Chronic blood loss anemia & 9.1 & 0.001 \\
\hline Valvular disease & 8.5 & 0.059 \\
\hline Chronic pulmonary disease & 8.4 & 0.001 \\
\hline Diabetes, uncomplicated & 5.9 & 0.129 \\
\hline $\begin{array}{l}\text { Hypertension (combine uncomplicated } \\
\text { and complicated) }\end{array}$ & 5.5 & 0.001 \\
\hline
\end{tabular}

${ }^{*} p$ value $<0.05$ statistical significance for the range. seen in those $>91$ years old $(p<0.001)$.

\subsection{Univariate and Multivariate Analysis}

Univariate analysis identified several patient factors which impacted mortality rates. (Table 7) Specifically, mortality rates were significantly higher among patients $>90$ years $(20 \%)(p=0.001)$, male patients $(7.3 \%)(p=$ $0.002)$, Native Americans (10.2\%) $(p=0.015)$, those having a gastrectomy for benign disease $(8 \%)(p=0.001)$, and patients with non-elective admission status prior to gastrectomy $(11.4 \%)(p=0.001)$. In addition, the presence of several co-morbidities also increased mortality rates including coagulopathy (25.2\%), renal failure (19.3\%), congestive heart failure (19.1\%) and fluid and electrolyte imbalances (13\%). On multivariate analysis (Table 8), age did not independently impact mortality and only non-elective admission $(p=0.001)$, hypertension $(p=0.001)$, valvular heart disease $(p=0.001)$, anemia $(p=0.001)$, and gastric cancer $(p=0.001)$ increased in-hospital mortality in patients undergoing gastrectomy.

\section{Discussion}

Today approximately 21,000 Americans are diagnosed annually with gastric cancer and half will die due to the lethality of the disease. It has been predicted that the proportion of the US population over 80 years old is expected to double in the next two decades going from $5 \%$ (1990) to $10 \%$ (2030) [6]. In addition, Americans 65 to 79 years who constituted $9.3 \%$ of the population in 2000 , are projected to increase to $12.5 \%$ by 2050 and $12.9 \%$ by 2070 [7]. Given current life table analysis, which predicts that patients who survive to the age of 80 - 85 years old will live another 8 years on average, the number of elderly patients ( $>80$ years) — and as a result the number of patients overall who will require gastric surgery-is increasing and will increase exponentially in the future $[8,9]$. Despite these facts, little is known about the clinical outcomes of gastrectomy for either benign or malignant conditions in elderly patients, as they are largely excluded from clinical trials given their presumed "high risk” status. More precisely, increased severity and frequency of co-morbidities, malnutrition and diminished organ reserve in the elderly and their demonstrable import on post-operative morbidity and mortality, has led to significant surgical bias towards performing less radical procedures or failure to consider the elderly for gastric surgery at all. Recently identified epidemiological shifts, from distal intestinal type to proximal diffuse type adenocarcinoma of the gastric cardia, most notably in the elderly patients and Western countries, may further complicate this picture $[10,11]$. At present, the best available data on the outcomes of gastric surgery in the elderly are 
A Population-Based Outcomes Analysis of the Impact of Age on Morbidity and Mortality Following

Gastrectomy: An Analysis of 13,799 Patients from the Nationwide Inpatient Sample Database

Table 8. Multi-variate analysis of in-hospital mortality for 13,799 patients undergoing total and partial gastrectomy (national inpatient sample database 2004-2008).

\begin{tabular}{|c|c|c|c|c|c|c|}
\hline \multirow{2}{*}{ Variable } & \multicolumn{2}{|c|}{ Overall } & \multicolumn{2}{|c|}{ Total gastrectomy } & \multicolumn{2}{|c|}{ Partial gastrectomy } \\
\hline & OR (95\% C.I.) & $p$ value & OR (95\% C.I.) & $p$ value & OR (95\% C.I.) & $p$ value ${ }^{*}$ \\
\hline Hypertension & $1.7(1.4-2.0)$ & 0.001 & $1.7(1.2-2.4)$ & 0.001 & $1.7(1.4-2.1)$ & 0.001 \\
\hline Valvular heart disease & $1.6(1.1-2.3)$ & 0.001 & & & $1.5(1.0-2.3)$ & 0.001 \\
\hline Anemia & $1.5(1.2-1.8)$ & 0.001 & & & $1.5(1.2-1.9)$ & 0.001 \\
\hline Non-elective admission & $2.4(2.0-2.9)$ & 0.001 & $2.7(1.9-3.7)$ & 0.001 & $2.4(1.9-3.1)$ & 0.001 \\
\hline Gastric cancer & $1.6(1.3-2.0)$ & 0.001 & $2.0(1.3-3.2)$ & 0.001 & $1.6(1.2-2.0)$ & 0.001 \\
\hline
\end{tabular}

Abbreviations: OR: odds ratio; C.I.: confidence interval; ${ }^{*} p$ value $<0.05$ statistical significance.

conflicting, lack inherent uniformity, and suffer primarily from the problem of small sample sizes and single institutional analysis [2,10,12-18].

Pisanu et al. evaluated 135 patients over 75 years old treated over an 11-year period, and determined that advanced age was not a contraindication to gastrectomy, nor was it predictive of increased morbidity and mortality (REF). These authors noted that the increased rate of co-morbidities and malnutrition were the primary factor contributing to increased mortality in the elderly rather than age alone. (REF) They also concluded that PG as opposed to TG was associated with improved quality of life (QOL) for this group, without sacrificing cure. Similar to these results Katai et al. noted significantly increased 30-day and 90-day mortality rates of 9.4\% and $18.8 \%$ in patients $>80$ years undergoing TG compared to those 50 - 69 years (1.4\% and 4.4\%, $p<0.001)$ [11]. Among patients $>80$ years old undergoing PG, mortality rates were $0 \%$ and $3.7 \%$ compared to $0.5 \%$ and $1.7 \%$ in patients 50 - 69 years of age ( $p=$ N.S). [14] Most notably, PG was also associated with an improved long-term survival compared to TG [19,20]. Sánchez-Bueno et al. also reported improved five-year survival among patients undergoing PG (48.1\%) compared to those undergoing TG $(18 \%)(p=0.001)$ [20]. However despite these encourage results, there remains no clear consensus on whether age alone independently affects morbidity and mortality after gastric surgery (and for that matter many surgeries). This is primarily due to the fact that most studies, in which increased mortality rates among the elderly have been reported, have failed to perform multivariate analysis allowing independent assessment of the contribution of advanced age [14]. In one of the few studies to perform multivariate analysis, Pisanu et al. reported a significantly higher mortality rate $17.2 \%$ following all types of gastrectomy in patients $>75$ years $(17.2 \%)$ compared to patients $<75$ years $(5.6 \%)$. However, they noted that several co-morbidities rather than age were the strongest predictors of increased post-operative mortality. Similar to the report by Pisanu et al., the current study also re- ports higher mortality rates with advancing age, but age by itself was not an independent predictor of mortality alone. Rather, our data supports the notion that being "old" is not nearly as important as being "sick and old" when it comes to mortality following gastric surgery. Much like a machine that continues to function well despite age, if given the proper maintenance (on or off warranty), the old patient with neglected health or chronic conditions is at greatest risk of failure (or death) when stressed. While the current study is certainly limited to drawing conclusions based on the variables assessed, it is noteworthy that the presence of gastric cancer, and preexisting hypertension, valvular disease, and anemia were all strongly associated with increased mortality. Of note, hypertension was the most prevalent co-morbid condition in those $>50$ years; while fluid and electrolyte imbalance was the most common co-morbidity in younger groups. These results are similar to those of Pisanu et al., who identified hypertension and stroke as the two most important factors affecting mortality following gastrectomy, as well as a report by Roviello et al. who noted that the two most common pre-existing conditions among patients undergoing surgery for gastric cancer were cardiovascular (57.7\%) and anemia/hypoproteinemia (41\%) $[21,22]$. The congruence of these findings across all three studies may be interpreted as implying that, whereas elderly patients are far more likely to have cardiovascular disease and suboptimal nutrition, particular attention to these co-morbidities, and optimization or remediation of these when possible, may result in decreased in-hospital mortality.

Several authors have reported that emergency surgery or non-elective gastric surgery (in addition to many other types of surgery) is associated with both higher morbidity and mortality rates $[21,23,24]$. In agreement with this literature, the current study also found that the elective or non-elective admission for gastrectomy correlated well with survival. Interestingly, the number of non-elective admissions for both TG and PG peaked at both ends of the age spectrum (youngest and oldest), with the in- 
creased number of non-elective admissions in the PG group compared to the TG group perhaps indicative of surgical bias and teaching, which urges performing the least radical procedure necessary in the sick patient.

Published reports of increased medical and surgical complications post gastrectomy among the elderly are numerous, and uniformly comment on decreased complications when performing PG compared to TG-albeit this is not just in the elderly population $[22,25,26]$. Gastrointestinal (GI) complications (11.3\%) were the most common surgical complication noted in the current study (ICD 997.4). Medical complications were noted in 16\% of patients, and were far more of a concern in TG patients (23.6\%) than PG patients (13.6\%). In the TG group, the most common complication overall was respiratory issues (18\%) which is similar to the findings of Bittner et al. [10]. While Bitner et al. did report on variations in complications between gastrectomy patients $<70$ and $>70$ years, the current report is the first study to analyze complications by decade. It is quite clear from the current results that the increased morbidity in elderly patients comes primarily in the form of medical complications (namely respiratory and cardiac events) and not surgical complications. (Tables 6(a) and (b)) Furthermore, cardiac events occurred at nearly twice the rate in the TG (6.1\%) compare that PG group (3.2\%). Taken on the whole, these results support the notion that when feasible, PG is the preferred surgical therapy in elderly patients (particularly in those with pre-existing co-morbidities that may increase the likelihood of post-operative complications).

The limitations of the study are that it is primarily retrospective in nature, as well as those factors inherent in working with large administrative databases. Complications that may have occurred post-discharge or on readmission within 30 days of procedure were not necessarily captured by the data set. That said, the underestimation of the actual complication rates should apply across all patients equally and thus not alter the findings of this report. In addition, the current study is limited by errors in coding that may have occurred at the time of the hospitalization. Under-reporting of co-morbidities in elderly patients is another potential limitation due to not including confounding factors like frailty, in which three or more of these following criteria are present: unintentional weight loss, exhaustion, weakness, slow walking speed, and low physical activity [27]. Finally, this study did not differentiate between stages of disease or extent of lymphadenectomy performed which could affect outcomes.

In summary, age alone is not an independent factor predictive of increased mortality following gastrectomy, while non-elective admissions, gastric cancer, hyperten- sion, valvular disease and anemia are. That said, whereas the elderly are more likely to be afflicted with these (and other co-morbidities), as well as to suffer from increased rates of post-operative morbidity, these results imply that surgical risk stratification of patients considered from gastrectomy, should focus primarily on the overall preoperative status of the patient's health rather than their chronologic age. Can gastrectomy be safely performed in elderly patients with acceptable clinical outcomes? The answer is yes. That said, all efforts should be made to optimize the patients remediable pre-operative co-morbidities prior to elective procedures, and provide appropriate counseling regarding the risks and expected postoperative course. Which type of gastrectomy is safer or preferred in the elderly patient remains a more difficult question to answer. While all surgeons have as their mantra "do no harm" and should select the least radical operation with similar outcomes and lowest anticipated morbidity/mortality, these goals must be undertaken against a backdrop of following sound surgical oncologic principles, by achieving a negative margin and adequate information to permit appropriate staging.

\section{REFERENCES}

[1] R. F. Saidi, J. L. Bell and P. S. Dudrick, "Surgical Resection for Gastric Cancer in Elderly Patients: Is There a Difference in Outcome?” Journal of Surgical Research, Vol. 118, No. 1, 2004, pp. 15-20. doi:10.1016/S0022-4804(03)00353-6

[2] K. Hanazaki, M. Wakabayashi, H. Sodeyama, et al., "Surgery for Gastric Cancer in Patients Older than 80 Years of Age,” Hepatogastroenterology, Vol. 45, No. 19, 1998, pp. 268-275.

[3] W. C. Meyers, R. J. Damiano Jr., F. S. Rotolo and R. W. Postlethwait, "Adenocarcinoma of the Stomach. Changing Patterns over the Last 4 Decades," Annals of Surgery, Vol. 205, No. 1, 1987, pp. 1-8. doi:10.1097/00000658-198701000-00001

[4] D. M. Parkin, P. Pisani and J. Ferlay, "Estimates of the Worldwide Incidence of Eighteen Major Cancers in 1985,” International Journal of Cancer, Vol. 54, No. 4, 1993, pp. 594-606. doi:10.1002/ijc.2910540413

[5] B. Martella, C. Militello, S. Spirch, et al., "Palliative Surgery for Gastric Cancer in Elderly Patients,” Acta BioMedica, Vol. 76, Suppl. 1, 2005, pp. 49-51.

[6] B. Cartier, "Carotid Surgery in Octogenarians: Why Not?" Annals of Vascular Surgery, Vol. 16, No. 6, 2002, pp. 751-755. doi:10.1007/s10016-001-0243-0

[7] United States Census Bureau, 2012. http://www.census.gov/population/www/projections/natd et-d1a.html

[8] I. Matsushita, H. Hanai, M. Kajimura, et al., "Should Gastric Cancer Patients more than 80 Years of Age Undergo Surgery? Comparison with Patients Not Treated 
Surgically Concerning Prognosis and Quality of Life,” Journal of Clinical Gastroenterology, Vol. 35, No. 1, 2002, pp. 29-34.

doi:10.1097/00004836-200207000-00008

[9] R. J. McKenna Sr., "Clinical Aspects of Cancer in the Elderly. Treatment Decisions, Treatment Choices, and FollowUp,” Cancer, Vol. 74, Suppl. 7, 1994, pp. 2107-2117. doi:10.1002/1097-0142(19941001)74:7+<2107::AID-CN CR2820741719>3.0.CO;2-1

[10] R. Bittner, M. Butters, M. Ulrich, S. Uppenbrink and H. G. Beger, "Total Gastrectomy. Updated Operative Mortality and Long-Term Survival with Particular Reference to Patients Older than 70 Years of Age," Annals of Surgery, Vol. 224, No. 1, 1996, pp. 37-42. doi:10.1097/00000658-199607000-00006

[11] P. Lauren, "The Two Histological Main Types of Gastric Carcinoma: Diffuse and So-Called Intestinal-Type Carcinoma. An Attempt at a Histo-Clinical Classification,” Acta Pathologica et Microbiologica Scandinavica, Vol. 64, No. 1, 1965, pp. 31-49.

[12] T. Bandoh, T. Isoyama and H. Toyoshima, "Total Gastrectomy for Gastric Cancer in the Elderly,” Surgery, Vol. 109, No. 2, 1991, pp. 136-142.

[13] S. Ishigami, S. Natsugoe, T. Saihara, et al., "Clinical and Pathologic Features of Early Gastric Cancer in Elderly Patients,” Hepatogastroenterology, Vol. 44, No. 16, 1997, pp. 1164-1168.

[14] H. Katai, M. Sasako, T. Sano and K. Maruyama, "The Outcome of Surgical Treatment for Gastric Carcinoma in the Elderly,” Japanese Journal of Clinical Oncology, Vol. 28, No. 2, 1998, pp. 112-115. doi:10.1093/jico/28.2.112

[15] K. Kitamura, T. Yamaguchi, H. Taniguchi et al., "Clinicopathological Characteristics of Gastric Cancer in the Elderly,” British Journal of Cancer, Vol. 73, No. 6, 1996, pp. 798-802. doi:10.1038/bjc.1996.139

[16] H. Kubota, T. Kotoh, D. K. Dhar, et al., "Gastric Resection in the Aged ( $>$ or $=80$ Years) with Gastric Carcinoma: A Multivariate Analysis of Prognostic Factors," Australian and New Zealand Journal of Surgery, Vol. 70, No. 4, 2000, pp. 254-257. doi:10.1046/j.1440-1622.2000.01802.x

[17] Y. Maehara, T. Oshiro, H. Oiwa, et al., "Gastric Carcinoma in Patients over 70 Years of Age," British Journal of Surgery, Vol. 82, No. 1, 1995, pp. 102-105. doi:10.1002/bjs.1800820134

[18] Y. Maehara, Y. Emi, S. Tomisaki, et al., “Age-Related Characteristics of Gastric Carcinoma in Young and Elderly Patients,” Cancer, Vol. 77, No. 9, 1996, pp. 1774-1780.
doi:10.1002/(SICI)1097-0142(19960501)77:9<1774::AID -CNCR3>3.0.CO;2-C

[19] H. J. Meyer, J. Jahne, H. Wilke and R. Pichlmayr, "Surgical Treatment of Gastric Cancer: Retrospective Survey of 1704 Operated Cases with Special Reference to Total Gastrectomy as the Operation of Choice," Seminars in Surgical Oncology, Vol. 7, No. 6, 1991, pp. 356-364. doi:10.1002/ssu.2980070607

[20] F. Sanchez-Bueno, J. A. Garcia-Marcilla, D. Perez-Flores, et al., "Prognostic Factors in a Series of 297 Patients with Gastric Adenocarcinoma Undergoing Surgical Resection,” British Journal of Surgery, Vol. 85, No. 2, 1998, pp 255-260. doi:10.1046/j.1365-2168.1998.00558.x

[21] F. Roviello, D. Marrelli, S. A. De, A. Messano, E. Pinto and A. Carli, "Complications after Surgery for Gastric Cancer in Patients Aged 80 Years and over," Japanese Journal of Clinical Oncology, Vol. 28, No. 2, 1998, pp. 116-122. doi:10.1093/jico/28.2.116

[22] A. Pisanu, A. Montisci, S. Piu and A. Uccheddu, "Curative Surgery for Gastric Cancer in the Elderly: Treatment Decisions, Surgical Morbidity, Mortality, Prognosis and Quality of Life,” Tumori, Vol. 93, No. 5, 2007, pp. 478484.

[23] A. G. Greenburg, R. P. Saik, J. J. Coyle and G. W. Peskin, "Mortality and Gastrointestinal Surgery in the Aged: Elective vs. Emergency Procedures," Archives of Surgery, Vol. 116, No. 6, 1981, pp. 788-791. doi:10.1001/archsurg.1981.01380180046009

[24] C. W. Wu, S. S. Lo, K. H. Shen, M. C. Hsieh, W. Y. Lui and F. K. P'eng, “Surgical Mortality, Survival, and Quality of Life after Resection for Gastric Cancer in the Elderly," World Journal of Surgery, Vol. 24, No. 4, 2000, pp. 465-472. doi:10.1007/s002689910074

[25] F. Bozzetti, "Total versus Subtotal Gastrectomy in Cancer of the Distal Stomach: Facts and Fantasy," European Journal of Surgical Oncology, Vol. 18, No. 6, 1992, pp. 572-579.

[26] J. Davies, D. Johnston, H. Sue-Ling, et al., "Total or Subtotal Gastrectomy for Gastric Carcinoma? A Study of Quality of Life,” World Journal of Surgery, Vol. 22, No. 10, 1998, pp. 1048-1055. doi:10.1007/s002689900515

[27] J. A. Sosa, P. J. Mehta, T. S. Wang, L. Boudourakis and S. A. Roman, "A Population-Based Study of Outcomes from Thyroidectomy in Aging Americans: At What Cost?" Journal of the American College of Surgeons, Vol. 206, No. 3, 2008, pp. 1097-1105. doi:10.1016/j.jamcollsurg.2007.11.023 